\title{
Off-Axially Arranged, Low Noise, High Collection Efficiency Ion Collector for Quadrupole Mass Spectrometer
}

\author{
FUSAFUMI NAKAO* AND TERUMASA YAMAMOTO*
}

(Received 3 October 1975)

\begin{abstract}
An improved ion detector for a partial pressure measurement at low levels has been developed using the off-axis technique. The ion detector consists of the electrostatic deflection plates and a channel electron multiplier. The arrangement and the configuration design are determined from the results of the map of electric equipotential surfaces and the trajectory analyses. The ion detector of this arrangement can greatly reduce the noise and base line shifts caused by photons and excited neutrals, which are dependent on the emission current and the pressures in the ion source. The noise count rate is on the order of $1-2 \mathrm{cps}$ at a multiplier voltage of 3000 volts. The ion collection efficiency of $100 \%$ in this detector arrangement is obtained at a multiplier voltage of 3000 volts or more with the help of electrostatic deflection plates. The ion detector also permits focusing of ions with fairly different ion energies onto the multiplier. The off-axis ion detector gives a factor of 140 increase in effective sensitivity of the mass spectrometer. The minimum detection limit of the mass spectrometer with the off-axis ion detector is also given.
\end{abstract}

\section{Introduction}

In the cource of the measurement of ion beams in mass spectrometer, a highly sensitive and low noise ion detector is often required and in some cases it needs to have a very fast response. Some methods of preliminary amplification of the ion beam using an open type secondary electron multiplier have been proposed by Allen," Smith, ${ }^{2)}$ Hereux and Hinteregger, ${ }^{3)}$ and Goodrich and Wiley. ${ }^{4}$ Methods using a scintillating screen have also been reported by Schutze and Bernhardt, ${ }^{5)}$ and Daly. ${ }^{6}$

With all these systems, currents of $10^{-14}$ amps, for example, can be amplified by up to $10^{6}$ with correspondingly improved response time making relatively fast recording feasible. The open type electron multiplier mounted in a high vacuum system may be generally used as an ion detector of mass spectrometer but noise of various kinds has been observed on the output signal. There is first of all the dark noise which is caused by the electron multiplier alone. This is due to high voltage vacuum breakdown phenomena in the high field regions around the electron multiplier or to surface breakdown along insulators. Another serious source of noise occurs when the electron multiplier is operated on a direct optical path with a mass spectrometer ion source as seen in a quadrupole mass spectrometer. This arrangement also causes the base line shifts with the pressure in the ion source or in the vacuum system. The basic causes of these noise and base line shifts are uncertain, but these are thought to be dependent on both emission current and pressure, i. e., it appears to be due to the photons (soft $\mathrm{X}$-rays and UV radiation) and excited neutrals passing directly from the ion source to the conversion dynode without being influenced by a

* Central Research Laboratories, Matsushita Electric Industrial Co., Ltd. 1006 Kadoma, Osaka, 571 Japan 


\section{F. Nakao and T. Yamamoto}

mass analyzing field. Since they have no charge, they follow a straight path or reflected path. The electron multiplier is generally very sensitive for these photons and particles, so that if the photons and particles impinge on the conversion dynode the corresponding dark current is detected on the electron multiplier output. An off-axis technique that is not affected by these undesirable interference signals would therefore be effective to increase the signal-to-noise ratio. Some attempts to block these undesirable signals have been made by many investigators. ${ }^{711}$ These methods are very excellent to block the undesirable signals originating from the ion source but the ion collection efficiency in their system is not thought to be very sufficient.

To solve these problems, we developed a new ion detector with low noise and highly ion collection efficiency without affecting by an emission current and a pressure in the ion source. A part of this ion detector has been described by the author elsewhere. ${ }^{(2)}$ This paper describes the detailed analysis and performance of the ion detector which is provided with a deformed electrostatic deflection plate and a channel electron multiplier. Also, we determine the minimum detectable ion current by this ion collector and give the minimum detection limit of the quadrupole mass spectrometer used here.

\section{Determination of the Ion Collector Configuration}

To prevent the direct incidence of undesirable photons and particles to the electron multiplier, it is necessary to provide a properly shaped optical block in front of the electron multiplier or to consider the setting arrangement of the electron multiplier. In order to obtain informations necessary in designing a configuration of ion collector, a measurement of the distribution of electric potential in several different configurations of ion collector were carried out by means of electric conducting sheet method ${ }^{13)}$ with an accuracy of several percent. By this method, the difficult determination of the potential field from the integration of Laplace's equation can be avoided. Details of this method are described in Appendix A. A scale model of the electrodes under investigation is painted on the electric conducting sheet by using a coloidal silver paint, and upon it are impressed voltages proportional to these of corresponding electrodes of the ion collector and the electron multiplier." The potential at any point on the electric conducting sheet is then proportional to that at the corresponding point in the investigated field so that a map of equipotential surfaces is easily obtainable.

Figure 1 shows an example of equipotential curves in some typical ion detector configurations. As seen from the results of the measurement of equipotential surfaces, the strength of the penetration field to the space between the exit aperture and the electron multiplier passing through an aperture of shield plate is strongly dependent on the multiplier voltage. In the configuration of the off-axis ion collector equipped with the deformed electrostatic deflection plates (Fig. 1 (a)), the field strength of the penetration field to the space between the deflection plates is depending upon the multiplier voltage and the deflection voltage, especially, the field gradient around the exit aperture is subject to influence strongly by the deflection voltage. When no electrostatic deflection plate is provided (Fig. 1 (b)), the penetration field in the region between the exit aperture and the shield plate is rather weaker as compared with the former case but the penetration field around the exit aperture spreads to the quadrupole rods region beyond the exit aperture. On the other hand, the aspect of the penetration field in an on-axis arrangement is very complicated 


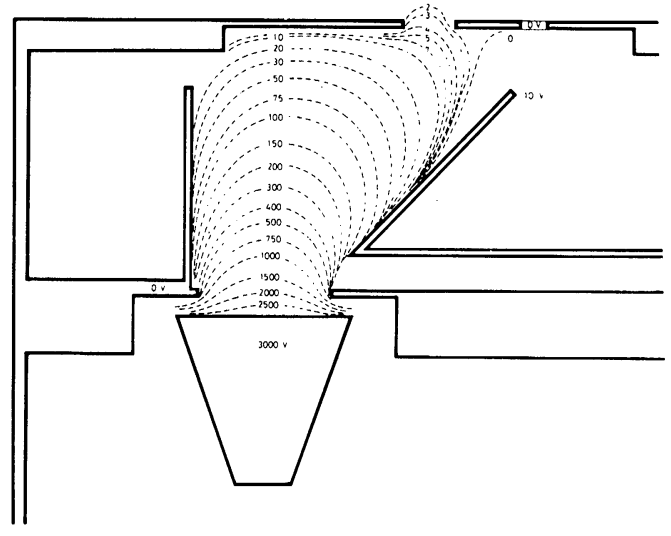

(a)

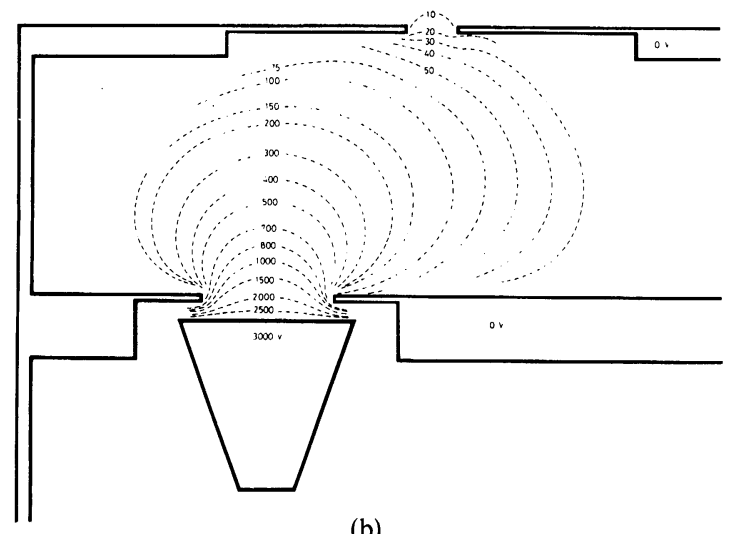

(b)

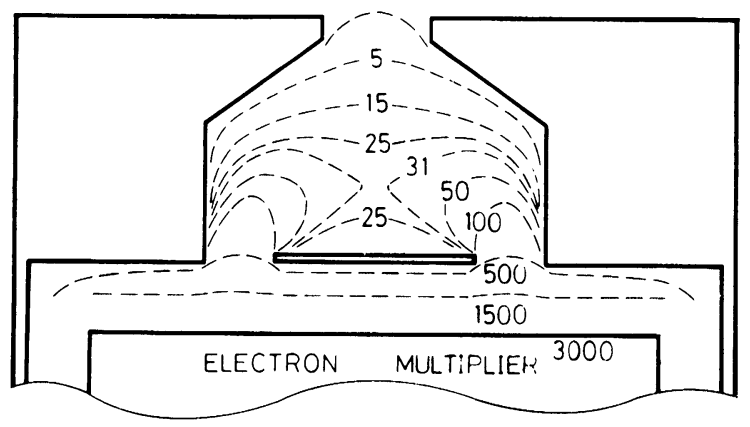

(c)

Fig. 1. A map of electric equipotential surfaces obtained from different types of ion detector configurations.

(a) the off-axis configuration with the deflection plates. multiplier voltage : 3000 volts, deflection voltage : 10 volts.

(b) the off-axis configuration without the deflection plates. multiplier voltage: 3000 volts.

(c) the on-axis configuration with the photon blind plate."' as shown in Fig. 1 (c). This arrangement is believed to be effective method to block photons and particles, ${ }^{7}$.11 but it is always accompanied by the ion losses because an ion trajectory around the exit aperture and the disk plate is much more subject to influence by the energy of ions incident into this region.

Ion trajectories in the region from the exit aperture to the electron multiplier were obtained graphically by the circle method ${ }^{15,20)}$ from the map of equipotential lines as shown in Appendix B. Because the energy of ions transmitted by a quadrupole mass filter lays in the energy range from 5.5 to $7 \mathrm{eV},{ }^{16)}$ the ray tracing is made by considering this ion energy. Also, we presumed that an angle of incidence of ions is $\pm 10^{\circ}$ with respect to the optical axis. Results obtained are shown in Fig. 2. It is found that, in the configuration of the off-axis ion collector equipped with the electrostatic deflection plates, ions started from a certain definite region of the exit aperture are focussed well electrostatically upon the electron multiplier. Also, it is shown that the trajectories of ions started from the same position on the exit aperture vary according to the energy of incident ions, the multiplier voltage, and the deflection voltage. It is also found that the landing position on the cone electrode is almost the same in spite of the ion energy and an angle of incidence. This means that the secondary electron yield from the electrode does not dependent on an angle of incidence. On the contrary, when the electrostatic plate is not provided, the ion trajectories are too much different from the former case, i. e., the ion trajectory depicts the sharp curve near the shield plate and reach to the cone electrode with an different angle of incidence. This suggest that when an angle of incidence of the incident ion is different from our case, incident ions are unable to reach the electron multiplier. 


\section{F. Nakao and T. Yamamoto}
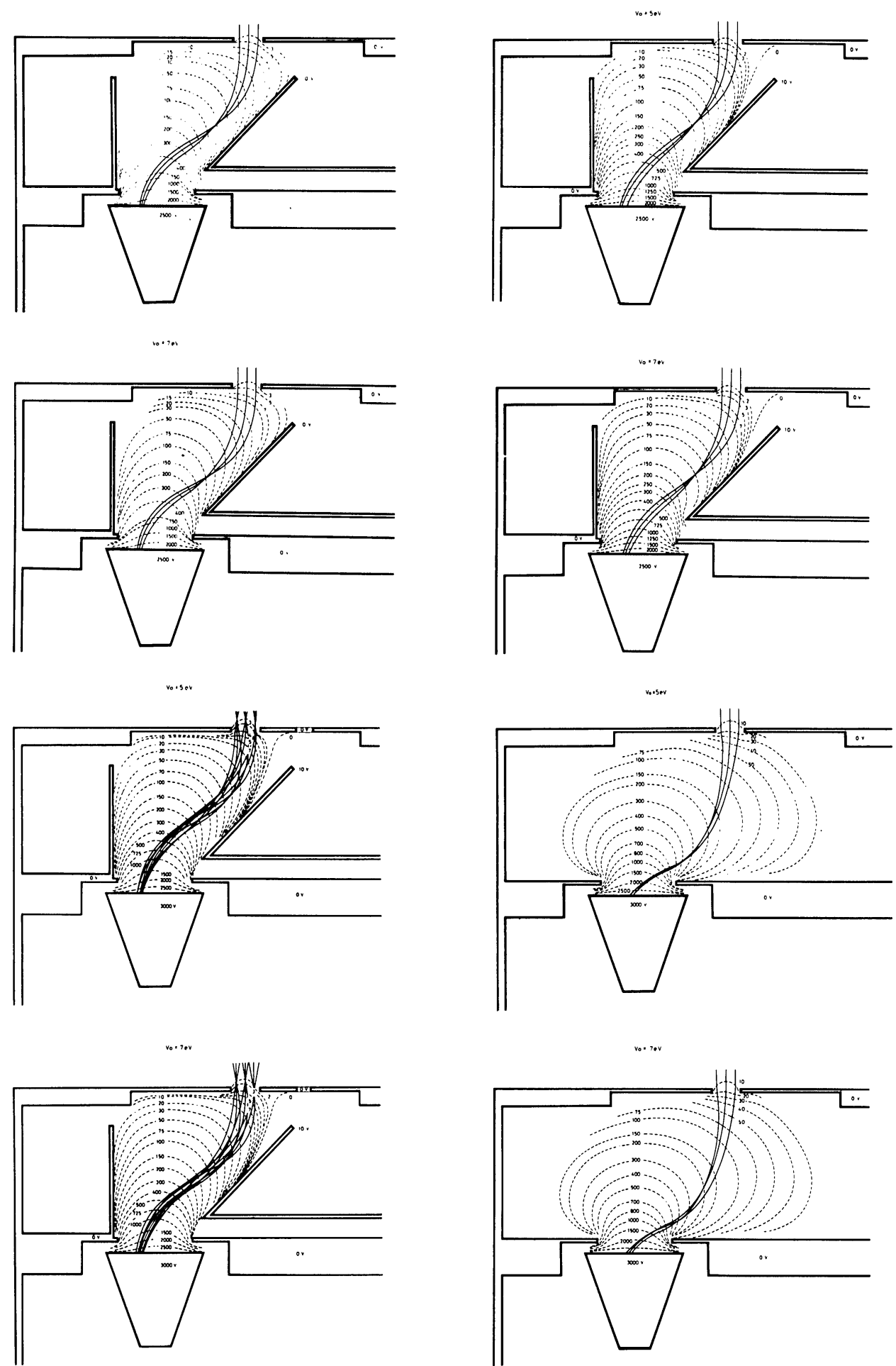

Fig. 2. Ion trajectory pattern in the space between the exit aperture and the deflection plate. Initial energy of ions incident into the space 1. presumed as $5 \mathrm{eV}$ and $7 \mathrm{eV}$, respectively. The angle of incidence of ions is also presumed as $\pm 10^{\circ}$ with respect to the optical axis (only the left hand side). 


\section{Procedure}

A main part of the ion collector with the off-axis mounting consists of the electrostatic deflection plates and a channel electron multiplier. The channel electron multiplier was chosen over the other types of electron multipliers because its structural dimensions and configurations were small and simpler. It also easily permits the off-axis mounting. The cross sectional view of the ion detector is schematically illustrated in Fig. 3. This arrangement with the electrostatic deflection plate was determined from the results of potential distribution plottings and the trajectory analyses as described in Sec. 2 in order that all the ions incident to the deflector space may be guided to the channel electron multiplier. The channel electron multiplier used is a Bendix channeltron multiplier type SEM-4219-X, ${ }^{17)}$ and it is located at a position separated from the optical axis of

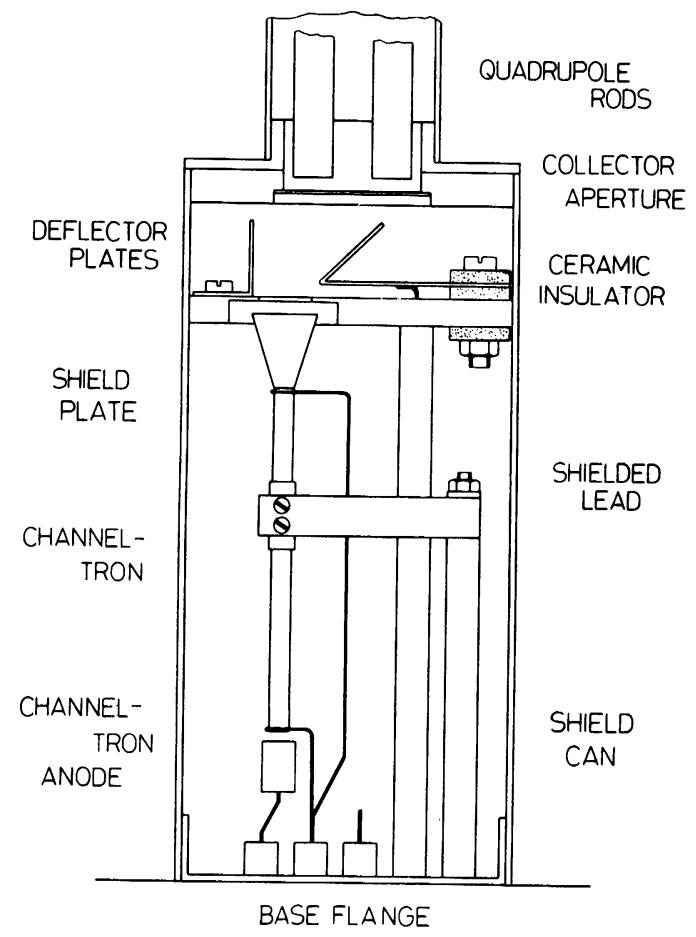

Fig. 3. Design details for improved ion collector with the channel electron multiplier. the mass spectrometer by a distance of $10 \mathrm{~mm}$. A multiplier shield plate with an aperture of $8 \mathrm{~mm}$ in diam is mounted in front of the cone of the multiplier at a distance of $2 \mathrm{~mm}$. One of the deflection plates is placed at an angle of $45^{\circ}$ with respect to the optical axis on the shield plate spaced $2 \mathrm{~mm}$ apart by ceramic insulators. This deflection plate is provided with a separately shielded lead to the outside of the vacuum system, and also can be used as an efficient Faraday collector. The other was directly placed perpendicularly to the shield plate, $15 \mathrm{~mm}$ from the optical axis. All the deflection plates are plated with gold to minimize the secondary electron emission by ions and photons impinging upon the deflection plate. The distance between the deflection plates and the exit aperture is $2 \mathrm{~mm}$. All of the electrodes and the multiplier are surrounded with a shield can in order to prevent the impingement of photons and particles upon the multiplier.

To measure the suppression effects for the photons and particles, a standard EAI 150A mass filter ${ }^{18)}$ was chosen for the experiments, but the ion detector was replaced with a new one as shown in Fig. 3. The multiplier anode was connected to a fast response picoammeter input through $50 \mathrm{~cm}$ of $\mathrm{RG} 3 \mathrm{C}-2 \mathrm{~V}$ cable.

Noise were measured with a National/ Panasonic VP-526A oscilloscope and base line shifts were measured with a National/Panasonic VP-654A strip chart recorder.

\section{Results and Discussion}

The operating performance of electrostatic deflection plate shown in Fig. 3 may be studied in more detail prior to the measurement of the suppression effects by plotting the output current as a function of the applied deflector voltage for several values of multiplier voltage. As can be seen from the results shown in Figs. 1 and 2, the field strength of the penetration field to the space between the deflection 
plates depends on the applied voltage to the multiplier. If the multiplier voltage is relatively low, the ion trajectories nearby the exit aperture is strongly jnfluenced by the deflection voltage. The plot of the output current versus the deflection voltage that is obtained at an argon pressure of $1.25 \times 10^{-6}$ Torr is shown in Fig. 4. The output current at a deflection voltage of 10 volts is up to 30 times as large as that obtained without applying the deflection

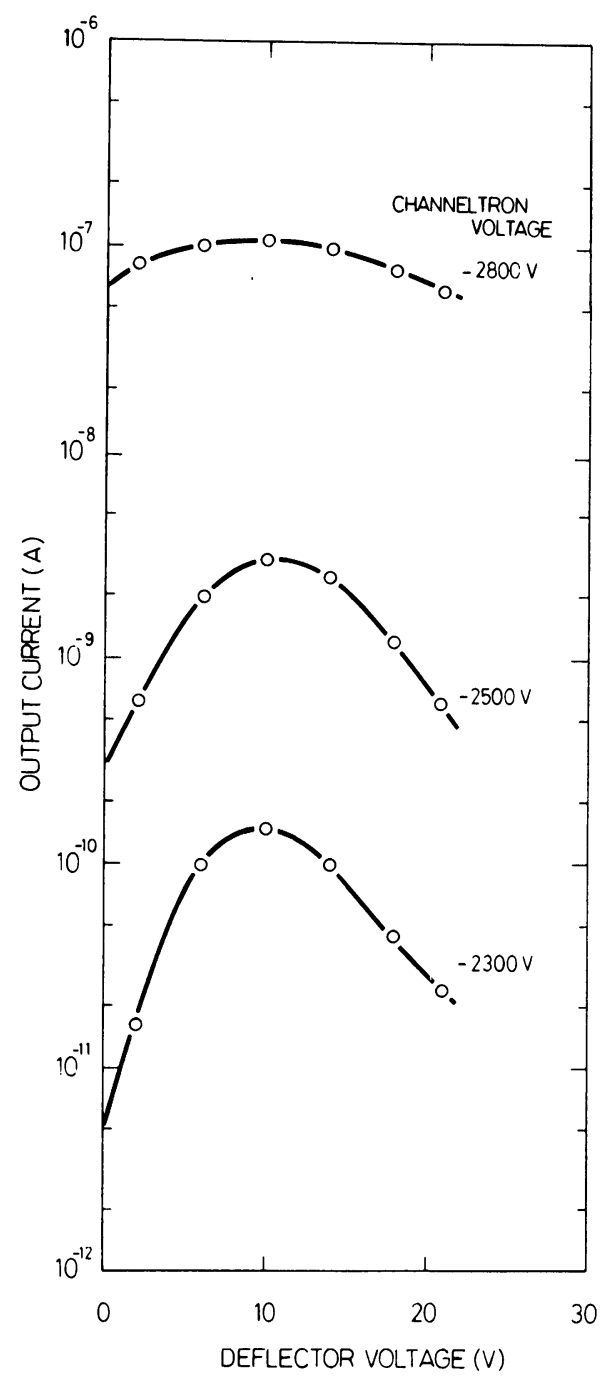

Fig. 4. Output current as a function of voltage on the deflection plate for various voltages of the channel electron multiplier. Argon : $P=1.25 \times 10^{-6}$ Torr ; $I e=1 \mathrm{~mA}$. voltage. It is concluded that, from this experimental facts, the effect of deflection plate for the case of low multiplier voltage is very large. For the high multiplier voltage, the penetration field spreads up to the exit aperture, and the deflection of the incident ions is controlled mainly by the penetration field. In the following experiments, the deflection voltage was maintained at 10 volts positive with respect to the grounded shield plate.

Figure 5 shows a plot of the ion collection efficiency as a function of the multiplier voltage of the ion detector with and without the deflection plates. With the deflection plates all of the incident ions to the deflector space may be guided to the multiplier with the help of the penetration field and the deflection plates. The collection efficiency of $100 \%$ in this arrangement is obtained at a multiplier voltage of 3000 volts

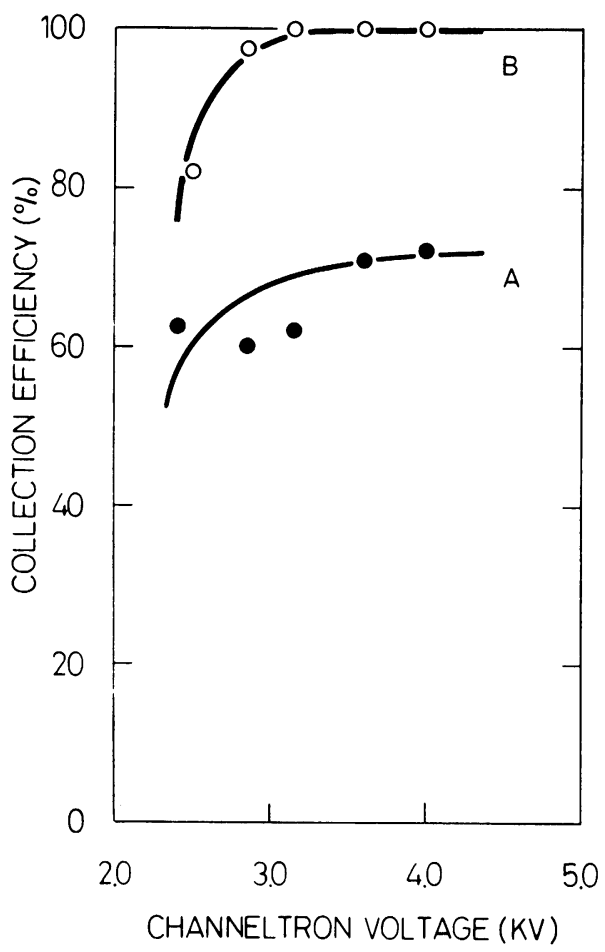

Fig. 5. A comparison of the ion collection efficiency obtained from the improved ion collector with (curve B) and without (curve A) deflection plates. Argon; $P=4 \times 10^{-6}$ Torr ; $I e=1 \mathrm{~mA}$. 
or more and a deflection voltage of 10 volts.

On the other hand, in the absence of the deflection plates it should be difficult to focus all the incident ions because the penetration field in the region of the exit aperture is not adequate, so several tens of percent of the total incident ions is lost to the shield plate and only the remaining 60 to $70 \%$ is collected through the aperture of shield plate onto the multiplier. This result agrees with that obtained from the hot cathode magnetron ionization gauge with the electron multiplier by Lafferty. ${ }^{19)}$ Thus a set of electrostatic deflection plates placed at the entrance of the conversion dynode is necessary for the enhancement of the collection efficiency of incident ions over a wide range of multiplier voltage.

In order to measure the noise and base line shifts, the multiplier was maintained at 3000 volts throughout the experiment. Also, the data were taken with an infinite resolving power operating condition of the mass spectrometer in order to prevent any gaseous ions from arriving at the multiplier. A dark output current caused by the light from the filament is fairly dependent on the emission current, i. e., the brightness of the filament. This is believed to be due to photons (soft X-rays and UV radiation) in the light. The dark output current is also dependent on the pressure in the ion source or in the vacuum system. Results obtained are shown in Fig. 6. As the filament temperature is raised to increase the emission current the dark output current starts to increase linearly. All the curves are parallel with a slope of $n=1.3$ in spite of the different ion detector configurations and operating pressures. The dark output current from the multiplier with the conventional ion detector (on-axis) is about 100 times larger than that of the off-axis ion detector. Such a large dark output current causes large base line shifts on the strip chart recorder, and this will also determine the

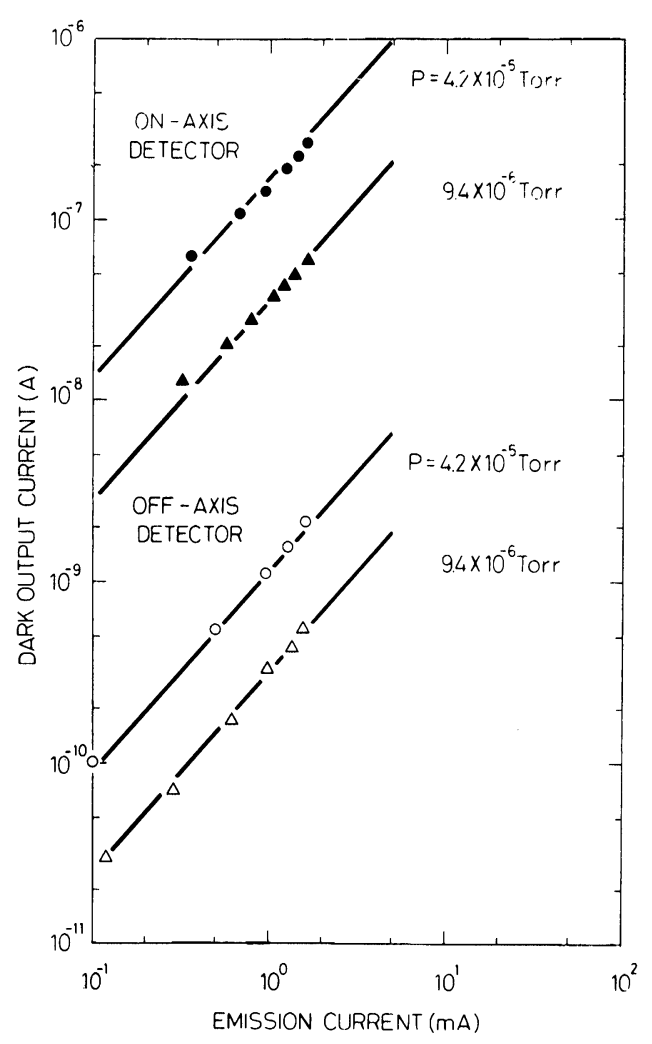

Fig. 6. Dark output current as a function of the emission currents. The dark output current also depends on the pressure in the vacuum system. All the curves are parallel with a slope of 1.3 .

minimum detection limit of the mass spectrometer. The light from the filament at normal operating temperatures (about $2300{ }^{\circ} \mathrm{K}$ ) in the conventional ion detector gives a noise of several thousand/ second, but in the off-axis detector the noise count rate is of the order of $1-2 \mathrm{cps}$ at the multiplier output. Figure 7 shows some typical aspect of resultant multiplier output current pluse obtained with on- and off-axis ion detector. These data were taken at a multiplier voltage of 3000 volts, a emission current of $1 \mathrm{~mA}$ and an argon pressure of $4 \times 10^{-7}$ Torr.

The dark output current due to the excited neutrals is thought to be attributable to the molecular density in the ion source or in the vacuum system. The dark output current in the on- and off-axis ion collector is shown in the 


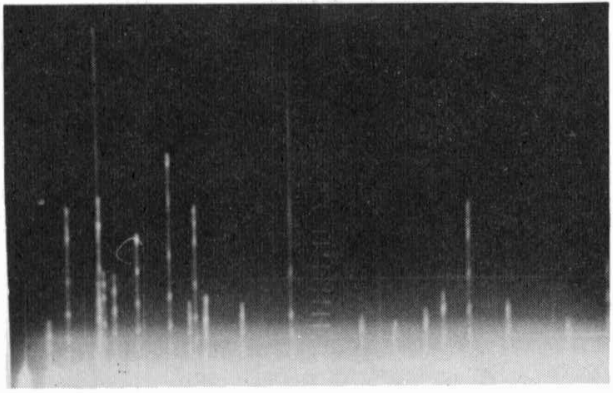

(a)

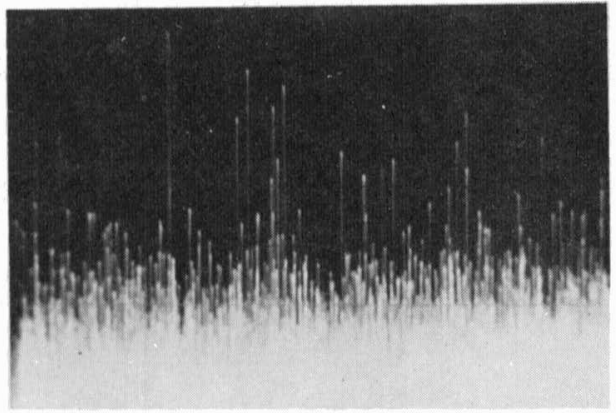

(b)

Fig. 7. Noise output current pulses from the channel electron multiplier obtained from on-axis (a) and off-axis (b) arrangement. multiplier voltage : 3000 volts, emission current $1 \mathrm{~mA}$, pressure $P=4 \times 10^{-7}$ Torr, scale $10 \mathrm{msec} /$ division, $50 \mathrm{mvolts} /$ division, exposure time: (a) $10 \mathrm{sec}$ and (b) 1 sec.

double logarithmic plot of Fig. 8. Data were taken at a emission current of $1 \mathrm{~mA}$ and with the infinite resolving power operating mode of the mass spectrometer. The dark output current is directly proportional to the pressure for a constant electron emission, and its magnitude in the conventional ion collector is much greater than that of the off-axis system. All the curves are approximate parallel with a slope of $n=1.1$ in spite of the different ion collector configurations. The off-axis ion collector configuration can be reduced the dark output current by a factor of 140 from that in the conventional one. From the results, the number of excited neutrals may be proportional to the density of gas molecules coming into the source. The off-axis ion collector with the electrostatic deflection plates can block not only the photons, but also the

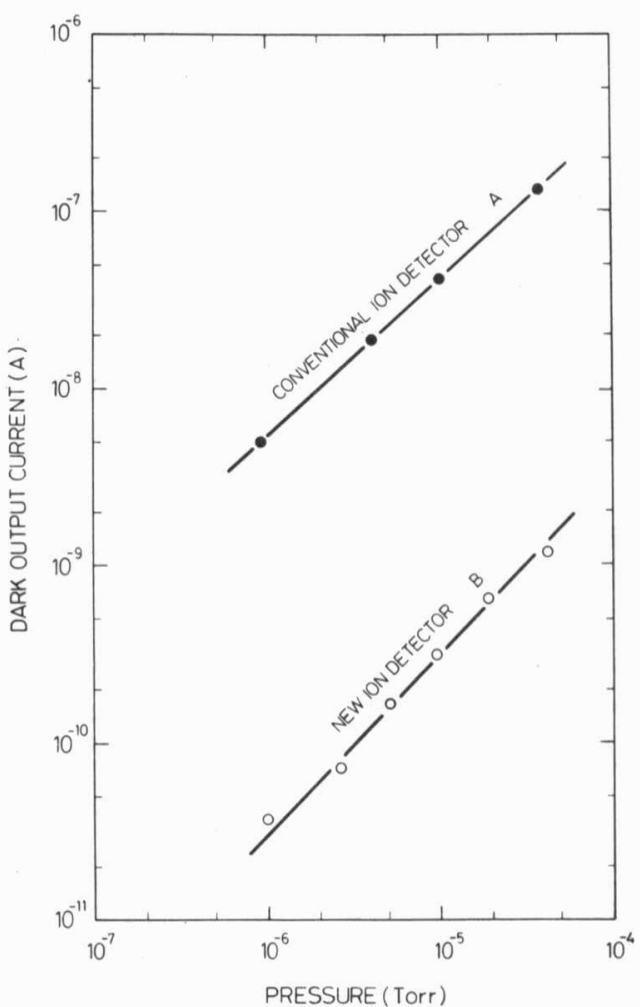

Fig. 8. Dark output current as a function of the pressure in the vacuum system. Curve $\mathrm{A}$ is obtained from the conventional (on-axis) ion collector and curve B is obtained from the off-axis ion collector. $I e=1 \mathrm{~mA}$.

excited neutrals. The number of pulses obtained per second as a function of the pressure in the ion source or in the vacuum system is also measured. The noise in the off-axis ion collector is much lower than that in the conventional one. The noise pulse count rate is almost the same as the former case for each collector.

As can be seen from the results shown in Figs. 6 and 8, the dark output current increases with increasing the emission currents, pressures, or both, and it starts to increase according to the equations

$$
I_{\mathrm{d}}=3.5 P^{\mathrm{L}} I_{\mathrm{e}}^{1.3} \quad \text { for the on-axis, }
$$

and

$$
I_{\mathrm{d}}=2.5 \times 10^{-2} P^{\mathrm{L}} I_{\mathrm{e}}^{1.3} \quad \text { for the off-axis. }
$$




\section{Off-Axially Arranged, Low Noise, High Collection Efficiency Ion Collector for Quadrupole Mass Spectrometer}

These equations will be of use in determining the minimum detection limit of the mass spectrometer by the difference of the ion collector configurations, i. e., the gain of multiplier at the multiplier voltage of 3000 volts is about $10^{6}$ so that an ion current of the order of $10^{-18} \mathrm{~A}$ can be detected by using the off-axis ion collector. This ion current corresponds to the partial pressure of $10^{-19}$ Torr, and a microimpurity of the order of $1 \mathrm{ppm}$ may be detectable without concentration of the sample to be analyzed.

\section{Conclusions}

The off-axis ion collector is an efficient technique to increase the signal-to-noise characteristics. By this technique, the noise count rate on the multiplier output can be reduced down to about one-thousand as compared with that of the conventional one. Preliminary experiments of determining the design of the ion collector by the electric conducting sheet method gave us several important informations. The electrostatic deflection plate constructed according to the results obtained from the electric conducting sheet method is shown sufficiently good ion collection efficiency over a wide range of multiplier voltage for ions incident to the deflection space. This construction design of the electrostatic deflection plate also permits a focus of ions with a fairly different ion energy onto the multiplier. Therefore, a highly ion collection efficiency is easily obtainable. This technique enables partial pressure measurements to be measured at low levels, and fast scanning of mass peaks become possible.

\section{Acknowledgement}

The authors would like to thank Dr. F. Oda of Central Research Laboratory for preparing the electric conducting sheets. Thanks are also due to Dr. S. Kisaka and K.
Iga of Central Research Laboratory for their continual encouragement and interest for this work.

\section{Appendix. A}

The electric conducting sheet method is based on the following principle:

From the concept of continuity of current in two dimensional field, provided the electric conducting sheet has a uniform resistance in all the directions, the equation

$$
\frac{\partial j_{\mathrm{x}}}{\partial x}+\frac{\partial j_{\mathrm{y}}}{\partial y}=0
$$

is statisfied. Here $j_{i}$ is the current density. If the specific conductivity of the electric conducting sheet is $\sigma$, then the current density is expressed by

$$
j_{\mathrm{x}}=\sigma E_{\mathrm{x}},
$$

and

$$
j_{\mathrm{y}}=\sigma E_{\mathrm{y}},
$$

respectively. Here $E_{\mathrm{i}}$ is the gradient of potential.

Setting $E_{\mathrm{i}}$ equal to

$$
E_{\mathrm{x}}=\frac{\partial \phi}{\partial x}
$$

and

$$
E_{\mathrm{y}}=\frac{\partial \phi}{\partial y}
$$

respectively, we obtain Laplace's equation as

$$
\frac{\partial^{2} \phi}{\partial x^{2}}+\frac{\partial^{2} \phi}{\partial y^{2}}=0 \text {. }
$$

Thus, it was found that the potential distribution in two dimensional electrode system, neglecting the space charge, was obtained by using the electric conducting sheet method.

\section{Appendix. B}

The problem of obtaining the charged particle trajectories on the basis of the map of 


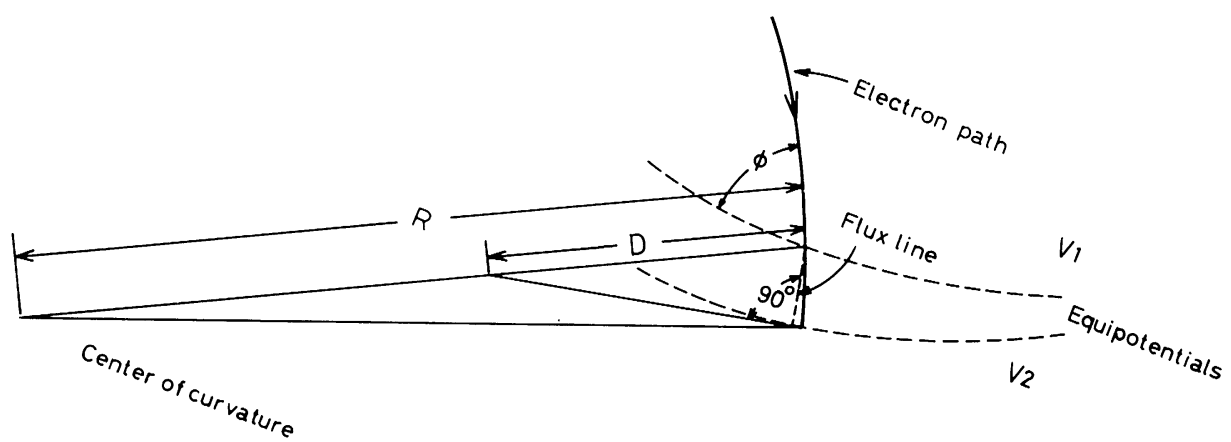

Fig. A-1. Procedure of the circle method.

equipotential lines can be solved by numerical integration. However, it is more expedient to make use of graphical methods which consist substantially in replacing the trajectory by segments of various simple curves such as circles, parabolas, etc. One of them, the circle method is based on a very simple relation between the radius of curvature of $R$ of the trajectory at any point $P$, the potential $V_{2}$ at $P_{1}$ and the component of the field $E_{\mathrm{r}}$ normal to the trajectory at $P$ (see Fig. A-1).

The energy principle for a charged particle whose initial energy is $\mathrm{eV}_{0}$, states that

$$
\frac{1}{2} m V^{2}=e\left(V_{2}+V_{0}\right)
$$

Since the centripetal acceleration of the charged particle is proportional to the radial force, we can write

$$
\frac{m V^{2}}{R}=e E_{\mathrm{r}}
$$

Eliminating $V$ between eqs. (B1) and (B2), we find that

$$
R=2\left(V_{2}+V_{0}\right) / E_{\mathrm{r}},
$$

which is basis of the graphical construction.

Suppose that the charged particle has arrived at the point $P$ lying on the equipotential
$V_{1}$ with the velocity $V$. The center $C$ of the curvature which will approximate the segment of path between the equipotential $V_{1}$ and $V_{2}$ will lie on the line normal to the charged particle path at $P$. The electric field at $P$ along a flux line is given by

$$
E=\left(V_{2}-V_{1}\right) / \mathrm{s},
$$

where distance $s$ is approximately the shortest distance between the equipotential lines $V_{1}$ and $V_{2}$. The component $E_{\mathrm{r}}$ is then

$$
E_{\mathrm{r}}=E \cos \varphi .
$$

Referring Fig. A-1, and substituting eqs. (B4) and (B5) to (B3), we obtain the radius of curvature as

$$
R=2\left[\left(V_{2}+V_{0}\right) /\left(V_{2}-V_{1}\right)\right] \cdot D .
$$

The distances $R$ and $D$ are laid off along a line perpendicular to the path at its crossing of the equipotential surface $V_{1}$. The distance is determined by the intersection of this line with a tangent to the equipotential $V_{2}$ so located that its point of tangency and the intersection of the charged particle path with equipotential $V_{1}$ lie on the same flux line.

It suffices now merely to multiply the distance $D$ by the known coefficient $2\left[\left(V_{2}+V_{0}\right)\right.$ $\left./\left(V_{2}-V_{1}\right)\right] \cdot D$ to find the position of $C$. The circular arc, whose center is at $C$, is the 


\section{Off-Axially Arranged, Low Noise, High Collection Efficiency Ion Collector for Quadrupole Mass Spectrometer}

desired approximation segment of path. By sufficient repetition of this procedure, the entire trajectory is graphically constructed.

\section{References}

1) J. S. Allen: Phys. Rev., 55, 336 (1939), 55, 966 (1939).

2) L. G. Smith: Rev. Sci. Instrum. 22, 166 (1951).

3) L. Heroux and H. E. Hinteregger: Rev. Sci. Instrum., 31, $280(1960)$.

4) G. W. Goodrich and W. C. Wiley: Rev. Sci. Instrum., 32, 846 (1961), 33, 761 (1962).

5) W. Schutze and F. Bernhard: Z. Physik. 161, 103 (1961).

6) N. R. Daly: Rev. Sci. Instrum., 31, 264 (1960).

7) B. E. Evans and W. Kneen : paper at 18 th Annual Conf. Mass Spect. Allied Topics. June 14-19 (1970), San Francisco, Calif., U. S. A.

8) U. T. I. Catalogue, D-0239 (1972)

9) A. Benninghoven and E. Loebach: Rev. Sci. Instrum., 42, 49 (1971).
10) J. E. Lester: Rev. Sci. Instrum., 41, 1513 (1970).

11) F. K. Truby and J. K. Rice: Rev. Sci. Instrum., 45, 854 (1974).

12) F. Nakao: Rev. Sci. Instrum., 46, No. 11 (1975). under printing.

13) I. Takeshita and F. Nakao: "Recent Developments in Mass Spectroscopy" p. 231 (University of Tokyo Press, 1970).

14) In our case, an applied voltage to the electrodes described on the electric conducting sheet is one-five hundred as large as the practical values.

15) V. K. Zworykin and J. A. Rajchman: Proc. Instrum. Radio Eng., 27, 558 (1939).

16) B. E. Evans and R. W. Supple: J. Vac. Sci. Technol., 8, 270 (1971).

17) Bendix Electro-Optics Div., Ann Arbor, Mich., 48107 U. S. A.

18) Electronics Associates Inc., Palo Alto, Calif., 94303 U. S. A.

19) J. M. Lafferty: Trans. 9 th Nat. Vac. Sympo., p. 438 (Pergamon Press, 1962).

20) W. W. Harman : "Fundamentals of Electronic Motion" p. 48 (McGrow-Hill Book Co., 1953) 